\title{
A novel spread slotted ALOHA based on cognitive radio for satellite communications system
}

\author{
Min Jia ${ }^{1 *}+$, Linfang Wang ${ }^{1+}$, Zhisheng Yin ${ }^{1}$, Qing Guo ${ }^{1}$ and Xuemai Gu ${ }^{1}$
}

\begin{abstract}
Cognitive radio (CR) as a promising way to solve the spectrum scarcity allows exploitation of the shared frequency bands while guaranteeing acceptable interference to incumbent users in satellite communication systems. An improved spread slotted ALOHA (SSA) based on multi-user, multi-channel CR model applying to the satellite communications is proposed in this paper. To make full use of the detection information of satellite earth stations, a novel joint collaborative sensing method is used in the sensing phase. Moreover, a better throughput is achieved by using the improved SSA strategy in the transmission phase comparing with the traditional slotted ALOHA (SA). Theoretical analysis shows that the system performs better when SSA is adopted. Theoretical analysis and simulation results indicate that the sensing method used in this model outperforms the traditional "hard combining" strategy in the whole sensing process.
\end{abstract}

Keywords: Cognitive radio, Satellite communication, Collaborative sensing, Multi-channel, SSA

\section{Introduction}

The demand for high-bandwidth application of mobile devices increases sharply with the boom in wireless communication, which needs the relevant technology to deal with the potential spectrum scarcity by enhancing the spectrum efficiency. One of the promising ways to improve spectrum efficiency is the cognitive radio (CR) proposed in [1]. The CR achieves the goal by allowing the secondary users (SUs) to access the unused licensed channels dynamically with a reliable energy detection performed by the SUs; therefore, the SUs will not disturb primary users (PUs).

The coexistence of PUs and SUs can be achieved by either a centralized way or a decentralized way. In the centralized strategy, a center cooperator and a control channel are necessary to sense the licensed channels collaboratively and to allocate the idle channels, respectively. Ref [2] studied a media access control (MAC) protocol, SCA-MAC, based on CSMA which made decisions on channels accessing with the statistics characteristic of

*Correspondence: jiamin@hit.edu.cn

${ }^{\dagger}$ Equal contributors

Communication Research Center, Harbin Institute of Technology, P.O.Box

3043, Yikuang Street 2, 150080 Harbin, China spectrum usage. Ganesan et al. in [3] proposed a sensing scheme which exploited the spatial diversity to improve the ability to sense in CR network. The throughput maximization and tradeoff in a centralized CR network are discussed in [4] and [5], respectively. The decision that the center cooperator makes on whether a licensed channel is idle can be carried out by hard combining or soft combining through the collaborative sensing. Hard combining, such as Ref [6], acts as the following process. To begin with, the SUs will determine whether a channel is idle through the energy detection and then send the result to a center cooperator. The cooperator will then make the final decision on the channel state based on all the SUs' results. In soft combining, e.g., Ref [7], each SU will send a full observation of signal energy on one channel to a cooperator and the cooperator will determine the channel state through these observations.

In the decentralized scheme (or distribute scheme), no cooperator or control channel exists. The SUs will privately perform channel sensing at some random or consecutive channels and utilize the channel which is detected to be idle to transmit data. Apparently, the reliable channel sensing is vital in the whole decentralized CR network. Refs [8-11] show a way, respectively, to 
permit the SUs to sense and access channel independently without central cooperators or control channels. The ALOHA-based CR network is discussed in $[12,13]$.

In recent years, the CR technology applied to satellite communications has been studied by researchers. Three application scenarios of CR technique in satellite communications were studied in [14], and the key challenges and enabling technologies for each scenario are also analyzed. A satellite-based multi-resolution compressive spectrum detection algorithm is proposed to achieve the coexistence of a mobile satellite system and an infrastructure wireless terrestrial network in [15]. Icolari in [16] proposed an energy detector based on radio environment mapping for the spectrum awareness functionality of a hybrid terrestrial/satellite scenario. An adaptive modulation scheme is proposed in [17] to mitigate the effect of rain on cognitive radio-based geostationary earth orbit (GEO) satellites which operate in the Ka-band.

An ALOHA based on CR model in the scenario of satellite communication which adopts the multi-user, multichannel CR model in [13] is proposed in this paper. And two major innovation points are as follows: (i) In the sensing phase, we adopt a novel collaborative sensing method proposed in [7] rather than a distributed method. By using this method, GEO satellite can make more reliable decisions and interfere with the PUs less. (ii) In the transmission phase, we bring spread slotted ALOHA (SSA) [18] in our work expecting better system performance. Besides, the collaborative sensing strategy in [6] is also introduced to make a contrast on which sensing scheme can perform better.

The rest of this paper is arranged as follows. The proposed model is described in Section 2. In Section 3, the throughput of the proposed model is analyzed, and the analytical expression of the throughput is also given. The simulation and analysis result as well as the contrast of the performance using different collaborative sensing methods are shown in Section 4. Finally, the conclusion is drawn in Section 5.

\section{System model description}

The CR model proposed in this paper will be discussed in the satellite communication scenario in [19]. As shown in Fig. 1, the GEO satellite and its satellite earth stations can be seen as the SUs and the fixed service stations are the PUs. Satellite earth stations communicate with the GEO satellite through cognitive links, i.e., the licensed channels. Satellite earth stations utilize the licensed channels only when they detect one channel as idle (i.e., no PU transmission in the channel), and the spectrum must be vacant if a PU accesses the licensed channel. It is clear to see in Fig. 1 that satellite earth stations may disturb the PUs because of imperfect channel sensing, so it is vital for satellite earth stations to perform continual spectrum sensing.
The cognitive satellite communication model is shown in Fig. 1. In this model, we consider a spectrum with $N$ licensed channels and the channels are distinguished by frequency. An example of channel state description is given in Fig. 2, where shaded areas represent the presence of the PU. The channel states will not change within one frame in this paper. Further, assume that the secondary users consist of $K$ satellite earth stations and one GEO satellite. Besides, the time axis in this model is divided into duration-fixed frame with the duration $T_{\mathrm{F}}$. Each frame consists three parts which are sensing time $T_{\mathrm{S}}$, report time $T_{\mathrm{R}}$, and transmission time $T_{\mathrm{T}}$. The concrete meaning of these three time types will be explained in the following part. The operating principle of this model can be described as two phases, which are the sensing phase and the transmission phase. The satellite earth stations will first detect the licensed channels in the sensing phase and then transmit data in the transmission phase through idle channels with spread slotted ALOHA algorithm. The two phases will be described then in order to reveal this model better.

\subsection{Sensing phase}

Each satellite earth station conducts channel sensing procedure during the sensing phase. As depicted in Fig. 3, $N_{\mathrm{S}}$ are sensing slots in which time lengths are $T_{\mathrm{Sm}}$ group sensing time, i.e., $N_{\mathrm{S}}=T_{\mathrm{S}} / T_{\mathrm{Sm}}$. In this model, each satellite earth station senses one licensed channel within a sensing slot and senses all licensed channels during the whole sensing time, which means $N_{\mathrm{S}}=N$. The channel detection can be achieved through energy detection which can improve the satellite earth stations' detection performance. Each satellite earth station obtains its full observation through energy detection and stores the signal samples of all licensed channels into its buffer after sensing time. Satellite earth stations will then send them to a GEO satellite. The GEO satellite will acquire the energy statistic of every licensed channel by summing the sample values received from all satellite earth stations with corresponding weights and finally reach a decision on states of all licensed channels. Then the GEO satellite will immediately broadcast the states of all the licensed channels to all satellite earth stations. Satellite earth stations transmit packets on idle channels with the result given by GEO satellites. However, if the GEO satellite wrongly declares a busy channel as an idle one, satellite earth stations may transmit packets on busy channels. As a result, satellite earth stations not only fail to transmit packets but also interfere with PUs. So, it is vital for GEO satellites to conduct accurate channel sensing. In the sensing phase of cognitive radio, the reliability of the decisions that GEO satellites make is subject to $P_{\mathrm{d}}$ and $P_{\mathrm{f}} . P_{\mathrm{d}}$ represents the probability of correct detection with the presence of PU, and $P_{\mathrm{f}}$ represents the probability of falsely declaring 


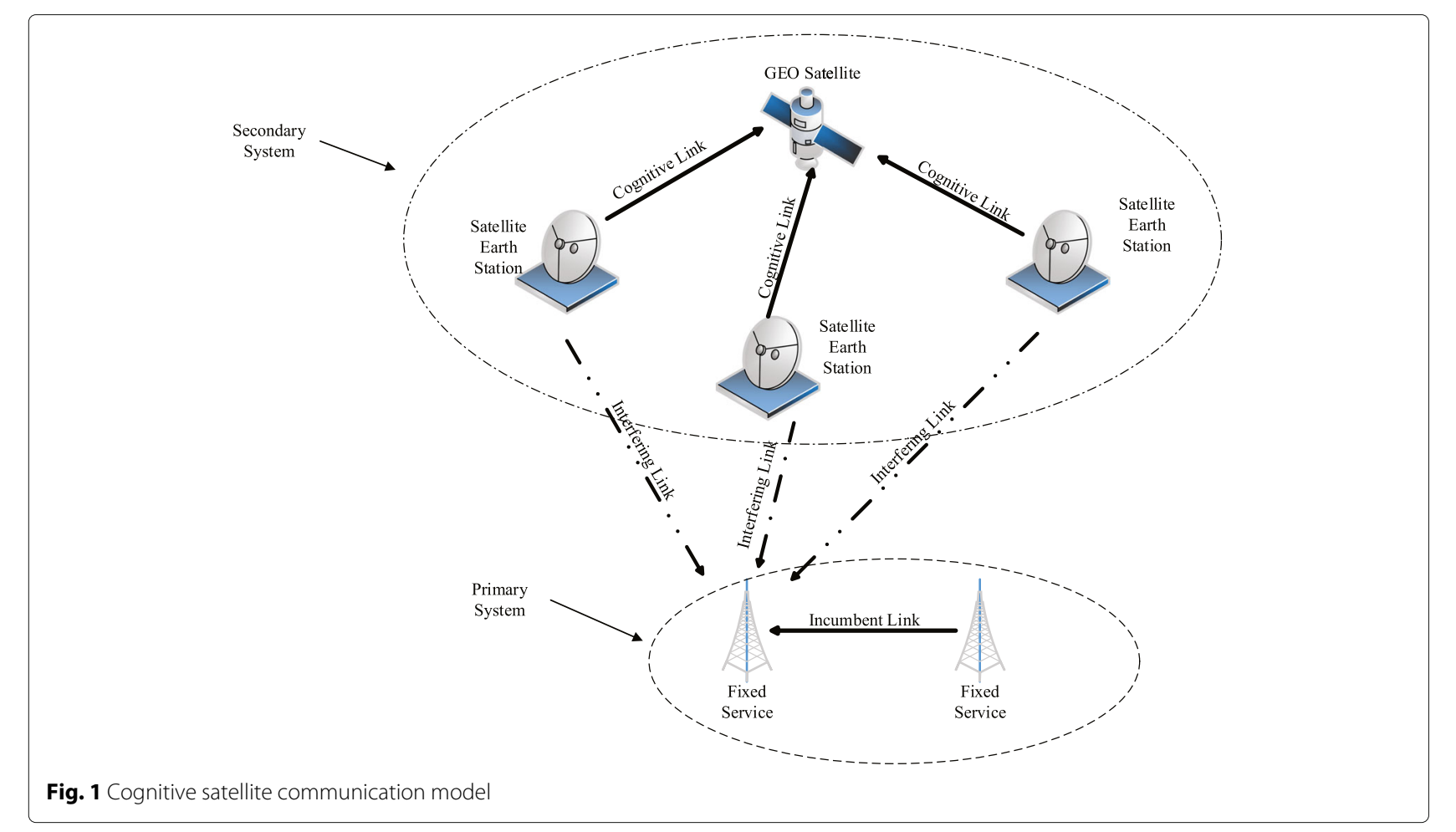

one vacant channel as busy. The whole sensing phase is illustrated in Fig. 4.

Other sensing methods can also help the GEO satellite make channel state decisions except the above scheme. A "hard combining" method is studied in [6], which is introduced to make a contrast with the sensing algorithm in this paper. The method can be stated as follows. Satellite earth stations will dependently reach a decision on the channel state and then send the results to GEO satellites. If all satellite earth stations claim idle on one channel, the satellite will announce the channel state vacant to all stations or the channel will be declared as a busy one.

\subsection{Transmission phase}

The SUs have to wait for the $T_{\mathrm{R}}$ (i.e., the report time), which includes propagation delay and processing delay, to receive the results of channel states after sensing phase, where $T_{\mathrm{R}}=\eta_{2} T_{\mathrm{Sm}}$. After obtaining the channel states, satellite earth stations will transmit packets using the SSA strategy in the transmission phase. In SSA, all transmission terminals are provided with a set of orthogonal codes, different terminals own the same code set, and the total number of the codes is represented by $N_{C}$. Considering the complexity of receivers, both the number of the codes and the spread factor (i.e., the length of a code) should be low. Besides, code words can be time-shifted versions of a single spreading-dispreading sequence [18]. The detail transmission processing can be described as follows. If one packet is generated, it will be first spread by a random spread code among the code set. Then a satellite earth station accesses an idle channel announced by a GEO satellite randomly at the beginning of a transmission slot

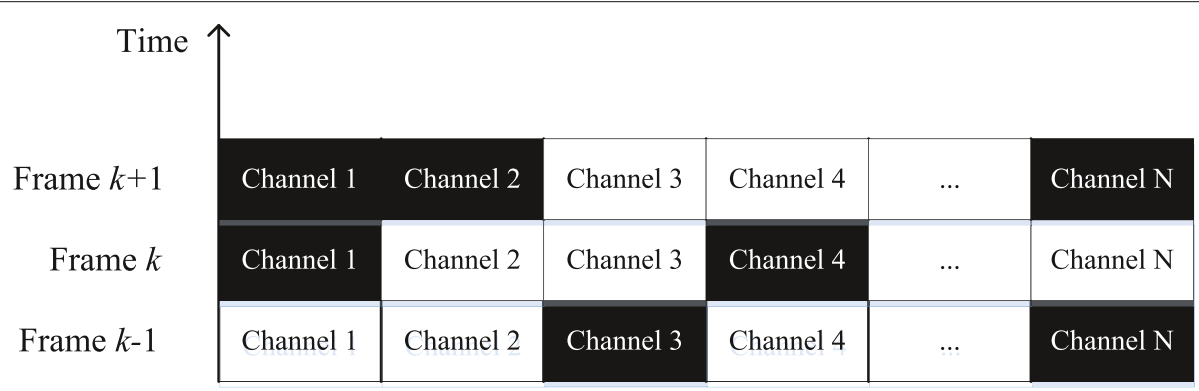

Fig. 2 Channel state in one frame 


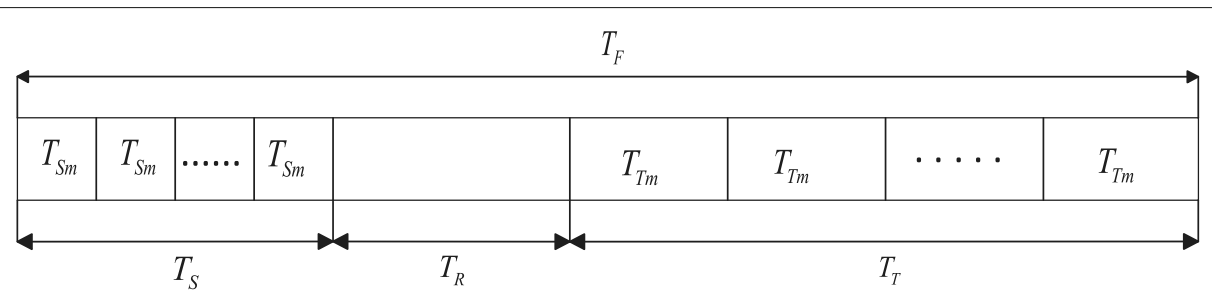

Fig. 3 Frame structure

$T_{\mathrm{Tm}}$ and sends a spread packet with a specific probability of $P_{\text {tra }}$, where $T_{\mathrm{Tm}}=\eta_{1} T_{\mathrm{Sm}}$. We can see from Fig. 3 that the transmission slot is the basic unit of transmission time and denotes that transmission time consists of $N_{\mathrm{T}}$ transmission slots, i.e., $N_{\mathrm{T}}=T_{\mathrm{T}} / T_{\mathrm{Tm}}$. Besides, suppose that the time length of a spread packet is equal to $T_{\mathrm{Tm}}$. It is obvious that more than one satellite earth station will transmit a packet with the same idle licensed channel, which means two or more packets may be overlapped in the same channel.

According to [18], we assume that the receiver of GEO satellite can only recover one packet on one channel. If the number of packets on one channel that arrived in a slot is more than one, only one of them can be processed and the rest of them will be seen as "interfering packets." The receiver can tolerate the interfering packets at a specific level; note that $N_{\mathrm{IL}}$ is the maximum number of interfering packets that the receiver can tolerate and once the receiver receives more than $N_{\mathrm{IL}}$ interfering packets, all the packets cannot be processed correctly. Further, the interfering packets will be declared as discarded. It is worth noting that the overlap is not equal to irreversible collision in SSA. The packet chosen by the receiver can be dispread and decoded correctly only if its spreading code is different from those used by all the other interfering packets. Figure 5 depicts the above workflow.

Based on the description of the transmission phase, if one packet is received successfully, except that it is sent to an idle channel, one of the following conditions should be satisfied: (1) No other satellite earth station sends packets on the same idle channel in the current slot; (2) Two or more satellite earth stations access the same idle channel and sends packets in the current transmission slot. Under this condition, if the number of packet interfered is no more than $N_{\text {IL }}$, one random packet will be processed by the receiver correctly only if the code word the packet uses is not the same with those the interfering packets use. The GEO satellite will broadcast the result on which packet is processed successfully. The satellite earth station will retransmit the packet if the packet is declared as a fail transmission. For analysis simplicity, the channel environment between satellite earth stations and the GEO satellite is set to be idle, which means the channel noise, channel gain, or near-far effect are not considered. Based on this, the packet is dispread and decoded with zero-error if the above conditions are satisfied.

\section{Numerical analysis}

In this section, the system throughput is formulated. Throughput is defined as the number of packets successfully transmitted in one sensing slot and will be the evaluation criterion of the model performance. The simple condition, which is that the satellite can give a perfect decision on the channel states and the number of the idle channels is fixed, is first considered. Then, we discuss how the throughput changes under the imperfect detection and the fixed idle channel. Finally, a more universal condition where every channel becomes busy with a specific

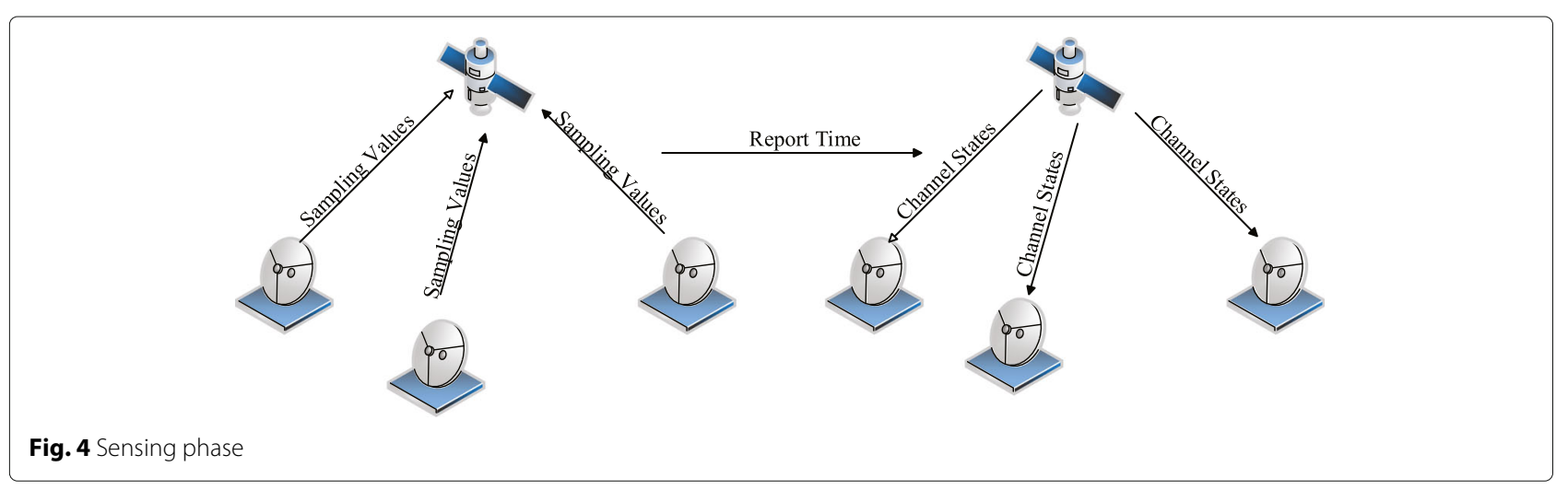




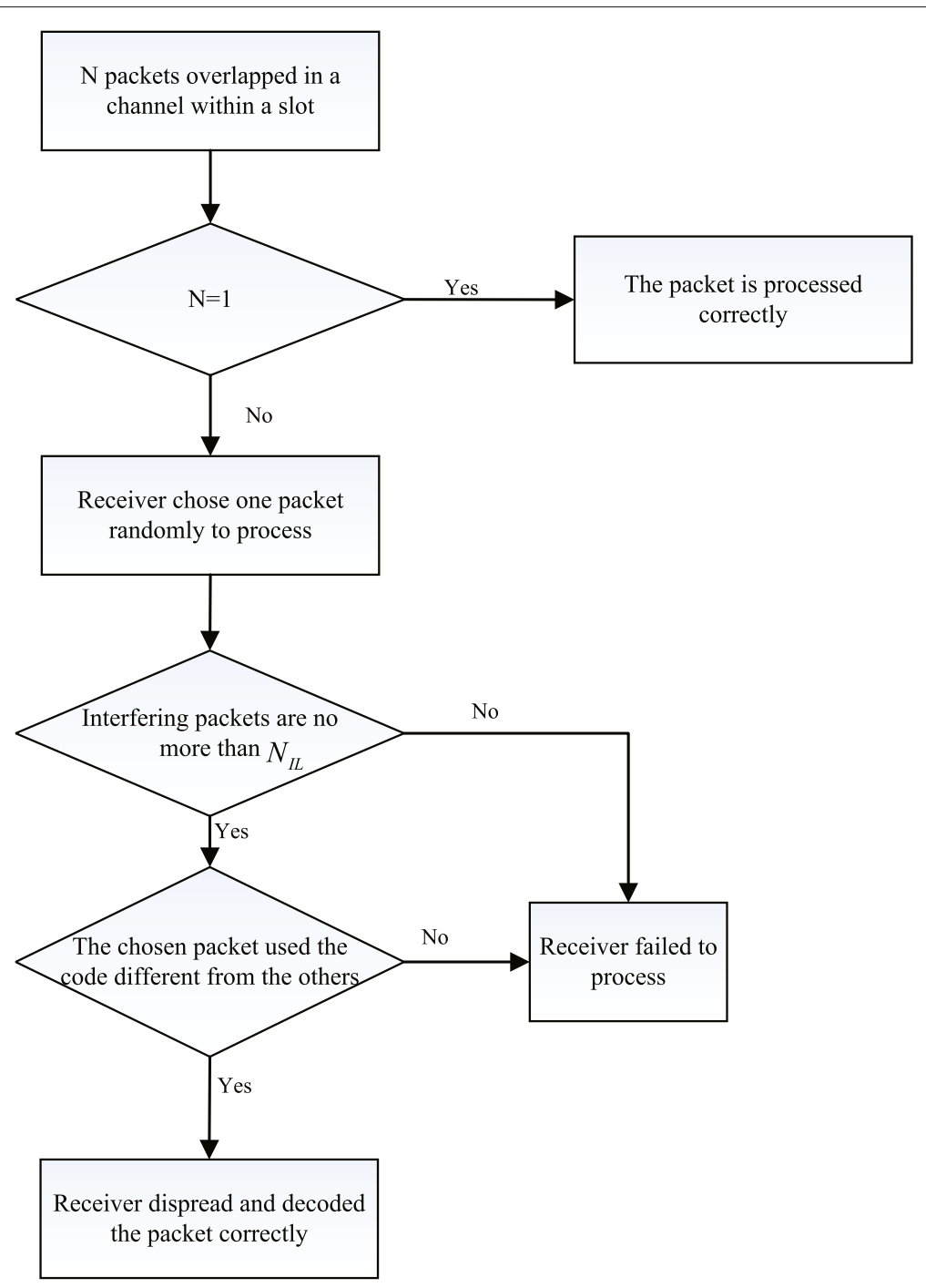

Fig. 5 Receiver's workflow in the transmission phase

probability in one frame is considered. In addition, it is assumed that there will be always at least one packet in the buffer of satellite earth station for simplicity as Ref [9].

For simplicity, we first suppose that there are always $M$ random idle channels among the entire licensed channels. Also, the GEO satellite can make the perfect decision on the channel states with the sampling values that the satellite earth stations send. In the transmission phase, the probability that the $k$ th satellite earth station chooses one of the $M$ channels and sends a spread packet within a slot is $P_{\text {access }}^{k}=P_{\text {tra }} / M$. Note that $P_{\text {access }}^{k}(k=1,2 \cdots K)$ is i.i.d, i.e., $P_{\text {access }}^{1}=P_{\text {access }}^{2}=$ $\cdots=P_{\text {access }}^{K}=P_{\text {access. }}$. It is obvious that, on the $r$ th channel, the probability that $m$ packets are overlapped within one transmission slot follows a binomial distribution. We denote the probability is represented by $P_{\text {rec }}^{r}(m)$ and $P_{\text {rec }}^{r}(m)=\left(\begin{array}{l}K \\ m\end{array}\right) P_{\text {access }}^{m}\left(1-P_{\text {access }}\right)^{K-m}$. As referred before, the receiver of GEO satellite can only endure a fixed number of interfering packets, and we assume the number is $N_{\mathrm{IL}}$ in this paper. We can formulate the probability that one packet can be successfully dispread and decoded among overlapped packets under the condition that interfering packets is less than $N_{\mathrm{IL}}$, i.e., $P_{\text {sur }}\left(m \mid m \leq N_{I L}+1\right)=\left(1-1 / N_{C}\right)^{m-1}$. Further, we can draw the conclusion that, on the $r$ th channel, the probability that $m\left(m \leq N_{I L}+1\right)$ packets overlapped and one of them is processed successfully is $P_{\text {suc }}^{r}\left(m \mid m \leq N_{I L}+1\right)=P_{\text {rec }}^{r}(m) P_{\text {sur }}(m)$. On the other hand, if the number of interfering packets is larger than $N_{\mathrm{IL}}$, the receiver will not dispread and decode 
any one of these packets correctly because of the overload interference. So, The average number of successful transmission on channel $r$ within one transmission slot, which is denoted as $S_{\text {ave }}^{r}=\sum_{i=1}^{N_{\mathrm{IL}}+1} P_{\mathrm{suc}}^{r}\left(i \mid i \leq N_{\mathrm{IL}}+1\right)$.

Further, we can formulate the average number of successful transmission packets on the entire $M$ channels within one transmission slot, which can be expressed as

$$
\begin{aligned}
& S_{\text {tot }}^{*}\left(P_{\text {tra }}, M\right)=\sum_{r=1}^{M} S_{\text {ave }}^{r}=M S_{\text {ave }}^{r} \\
& =M \sum_{j=1}^{N_{\mathrm{IL}}+1}\left(\begin{array}{c}
K \\
j
\end{array}\right)\left(\frac{P_{\text {tra }}}{M}\right)^{j}\left(1-\frac{P_{\text {tra }}}{M}\right)^{k-j}\left(1-\frac{1}{N_{\mathrm{C}}}\right)^{j-1}
\end{aligned}
$$

It is worth noting that the definition of throughput in this paper is the number of packets transmitted successfully in one sensing slot. Finally, we obtain the throughput of this simple scenario, which is represented by $S_{\text {sys }}^{*}\left(P_{\text {tra }}, M\right)$

$$
\begin{aligned}
S_{\text {sys }}^{*}\left(P_{\text {tra }}, M\right) & =\frac{T_{T}}{T_{\mathrm{S}}+T_{T}+T_{\mathrm{R}}} S_{\text {tot }}^{*}\left(P_{\text {tra }}, M\right) \\
& \left.=\frac{N_{T} \eta_{1}+N_{\mathrm{S}}+N_{T} \eta_{1}+\eta_{2}}{N_{\text {tot }}} S_{\text {tra }}^{*}, M\right)
\end{aligned} .
$$

Now we consider that the channel state decisions made by the GEO satellite is not perfect, which means $P_{d} \neq 1$ and $P_{f} \neq 0$. If a channel is idle, GEO satellite will detect this channel as idle with the probability of $1-P_{f}$. On the other hand, GEO satellite will claim a busy channel as idle with the probability of $1-P_{d}$. Any channel state decision made by GEO satellite is independent. Under the condition of fixed $M$ random idle channels, the average number of vacant channel that satellite broadcasts to satellite earth stations is $N_{\text {idle }}(M)=\left(\begin{array}{c}N \\ M\end{array}\right)\left(1-P_{\mathrm{f}}\right)+$ $\left(\begin{array}{c}N \\ N-M\end{array}\right)\left(1-P_{\mathrm{d}}\right)$. So, The system throughput with the imperfect detection is obtained as

$$
S_{\text {sys }}\left(P_{\text {tra }}, M\right)=\frac{\left(\begin{array}{c}
N \\
M
\end{array}\right)\left(1-P_{\mathrm{f}}\right)}{N_{\text {idle }}(M)} S_{\text {sys }}^{*}\left(P_{\text {tra }}, M\right) .
$$

The dynamic channel states are taken into account in this part. For analysis simplicity, we assume that channel state changes slowly so that the channel state will not change in one frame. We also assume that every channel will become vacant with the probability of $q$ in every frame. So, the probability that $M$ channels that are idle in one frame obeys binomial distribution. We denote the probability as $P_{\text {idle }}(M)$ and it can be expressed as follows.

$$
P_{\text {idle }}(M, q)=\left(\begin{array}{c}
N \\
M
\end{array}\right)(1-q)^{N-M} q^{M}
$$

Therefore, we can obtain the system throughput with imperfect detection and dynamic channel states as follows.

$$
\begin{aligned}
S_{\text {sys }}\left(P_{\text {tra }}, q\right) & =E\left[S_{\text {sys }}\left(M, P_{\text {tra }}\right)\right] \\
& =\sum_{m=1}^{N} S_{\text {sys }}\left(P_{\text {tra }}, m\right) P_{\text {idle }}(m, q)
\end{aligned}
$$

\section{Simulation results}

The simulation and analysis results are depicted in this section. The simulation is performed via Monte-Carlo simulation. In our simulation, the parameters are assigned as follows. The number of licensed channels $N=10$, the number of satellite earth stations $K=8$, the duration of sensing time slot $T_{\mathrm{Sm}}=2 \mathrm{~ms}, \eta_{1}=10, \eta_{2}=120$, and the number of transmission slot $N_{\mathrm{T}}=100$. We suppose that the sample frequency in sensing phase is $6 \mathrm{MHz}$, i.e., $f_{\mathrm{s}}=6 \mathrm{MHz}$. We obtain the relationship between $P_{\mathrm{f}}$ and $P_{\mathrm{d}}$ from [7], which can be expressed as follows.

$$
P_{\mathrm{f}}=Q\left(\sqrt{2 \Phi \xi+1} Q^{-1}\left(P_{\mathrm{d}}\right)\right)+\xi \sqrt{\frac{T_{\mathrm{Sm} f_{\mathrm{s}}}}{2}} \sum_{i=1}^{K} \omega_{i}\left|h_{i}\right|^{2}
$$

where $\xi$ means the ratio of signal to noise at the PU node, $h_{\mathrm{i}}$ means the channel gains and obeys the Gaussian distribution with mean zeros and variance one, and $\omega_{i}=$ $\left|h_{i}\right|^{2} / \sqrt{\sum_{i=1}^{K}\left|h_{i}\right|^{2}}$. Finally, we assume $N_{C}=5$ referring to Ref. [18]. The simulated and analytical results are shown in the following part. Figure 6 shows the system throughput with the change of $P_{\text {tra }}$ under the perfect detection and fixed number of idle channels. It can be seen that the simulated results are corresponding well with the analytical results at different $N_{\mathrm{IL}}$.

Figure 7 gives the simulation on system throughput with imperfect channel detection and fixed idle channel number, where the relevant parameters are defined as $\xi=$ $-15 \mathrm{~dB}$ and $P_{\mathrm{d}}=0.9$. From Figs. 6 and 7, when $N_{\mathrm{IL}}=1$, the throughput tendency with $P_{\text {tra }}$ increases first and then decreases. The reason is that with the increase of $P_{\text {tra }}$, the number of packets arriving in a slot increases and the throughput increases consequently. However, it also becomes more difficult for the receiver to dispread and decode the packets due to the continual increasing number of the packets arriving in a slot on one channel. At this point, the throughput decreases when $P_{\text {tra }}$ exceeds a specific level. In the scenario of $N_{\mathrm{IL}}=3$, throughput is improved as $P_{\text {tra }}$ increases because the interference level is always tolerated. In addition, we can see that, when $N_{\text {IL }}=0$, the SSA transmission model is equal to traditional slotted ALOHA. It is apparent that the system will 


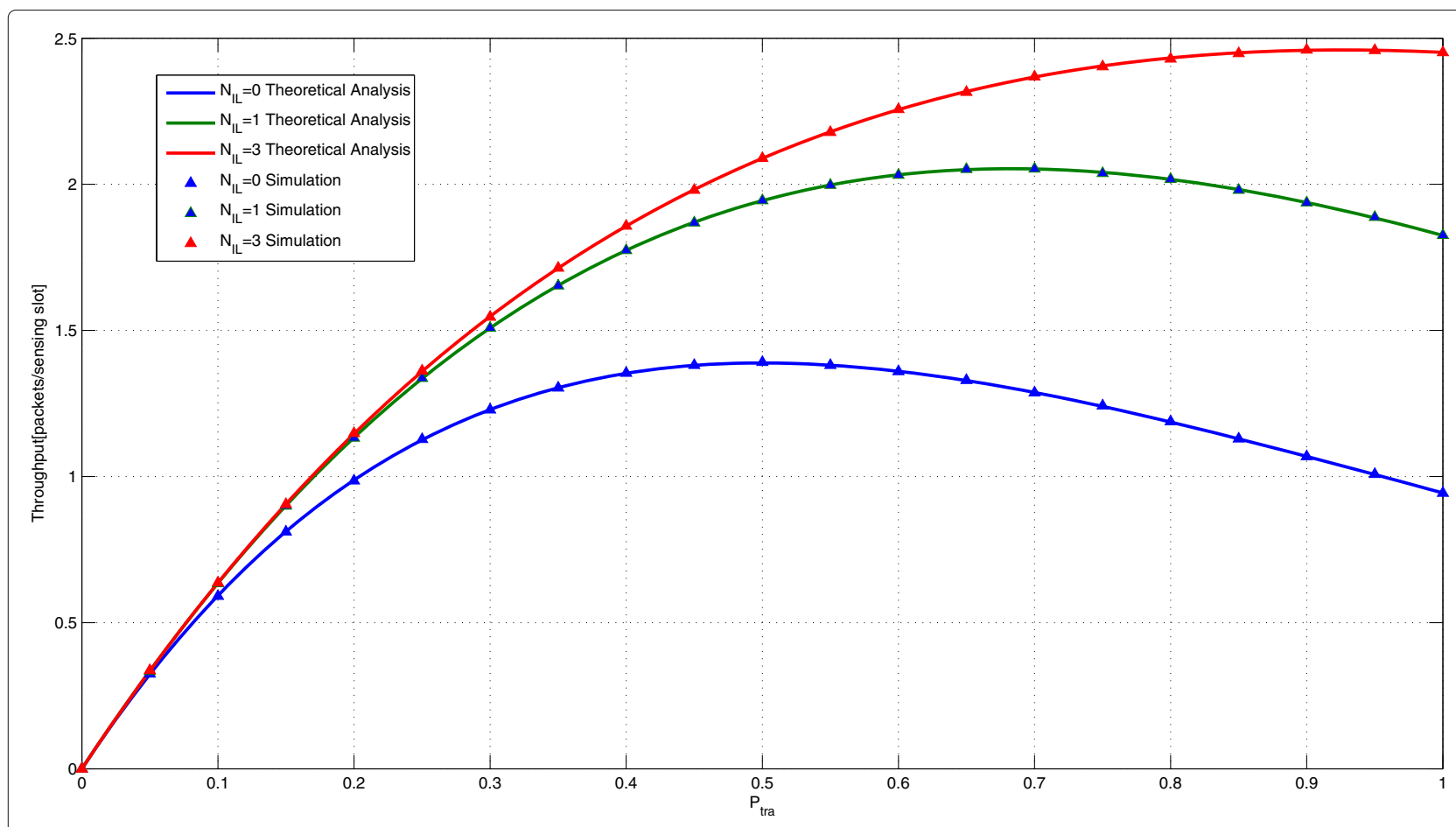

Fig. 6 Throughput with perfect detection

obtain more successful transmission when SSA strategy is adopted.

Figure 8 shows the simulation with imperfect channel detection and dynamic channel states; the relevant parameters are defined as $q=0.4, \xi=-15 \mathrm{~dB}$, and
$P_{\mathrm{d}}=0.9$. We also bring the "hard combining" sensing scheme, which is depicted in the preceding part in this paper, in our model to see which sensing algorithm performs better. We can see from Fig. 8 that the two sensing schemes perform closely in this model when $P_{\text {tra }}$ is

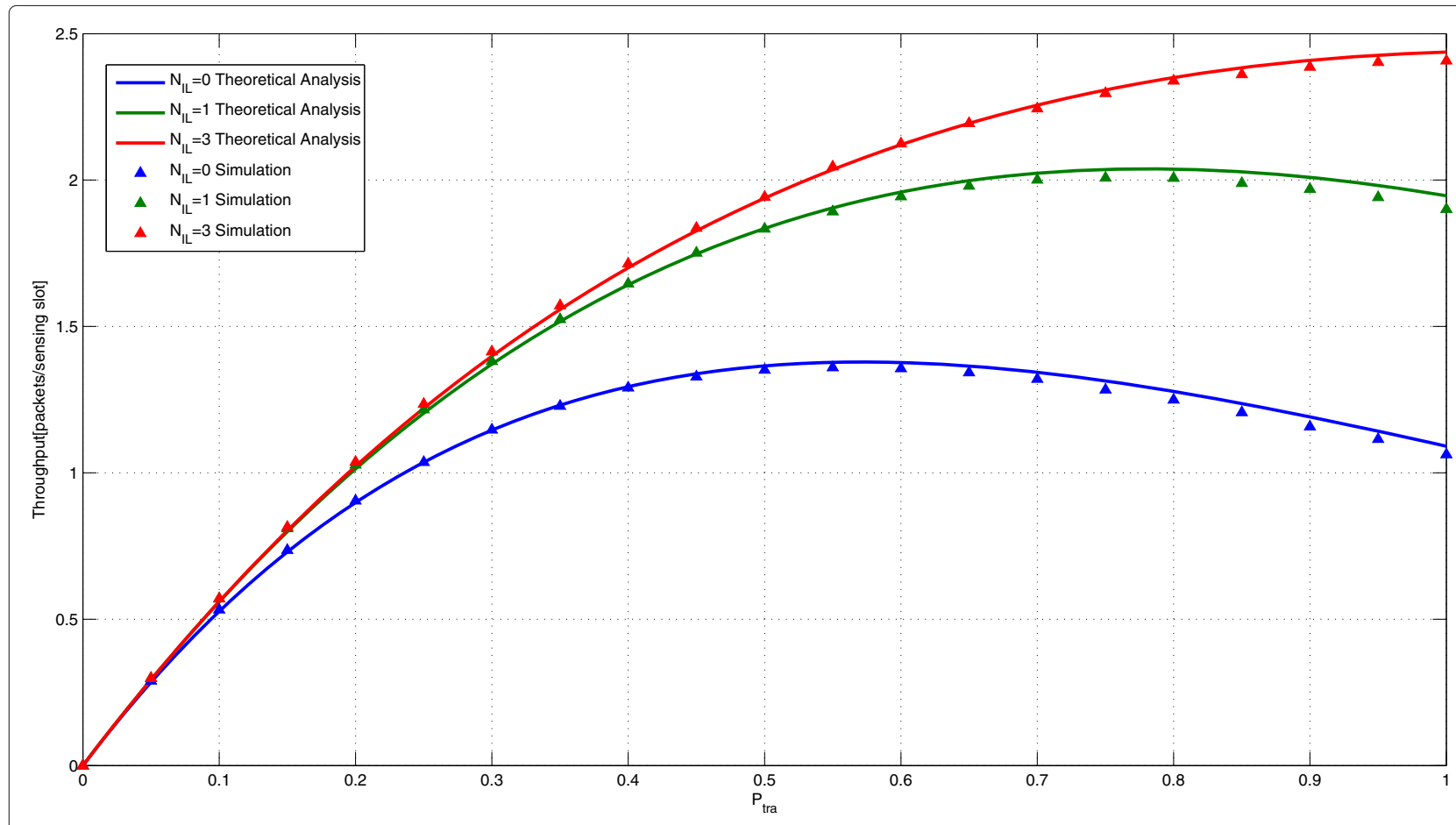

Fig. 7 Throughput with imperfect detection 


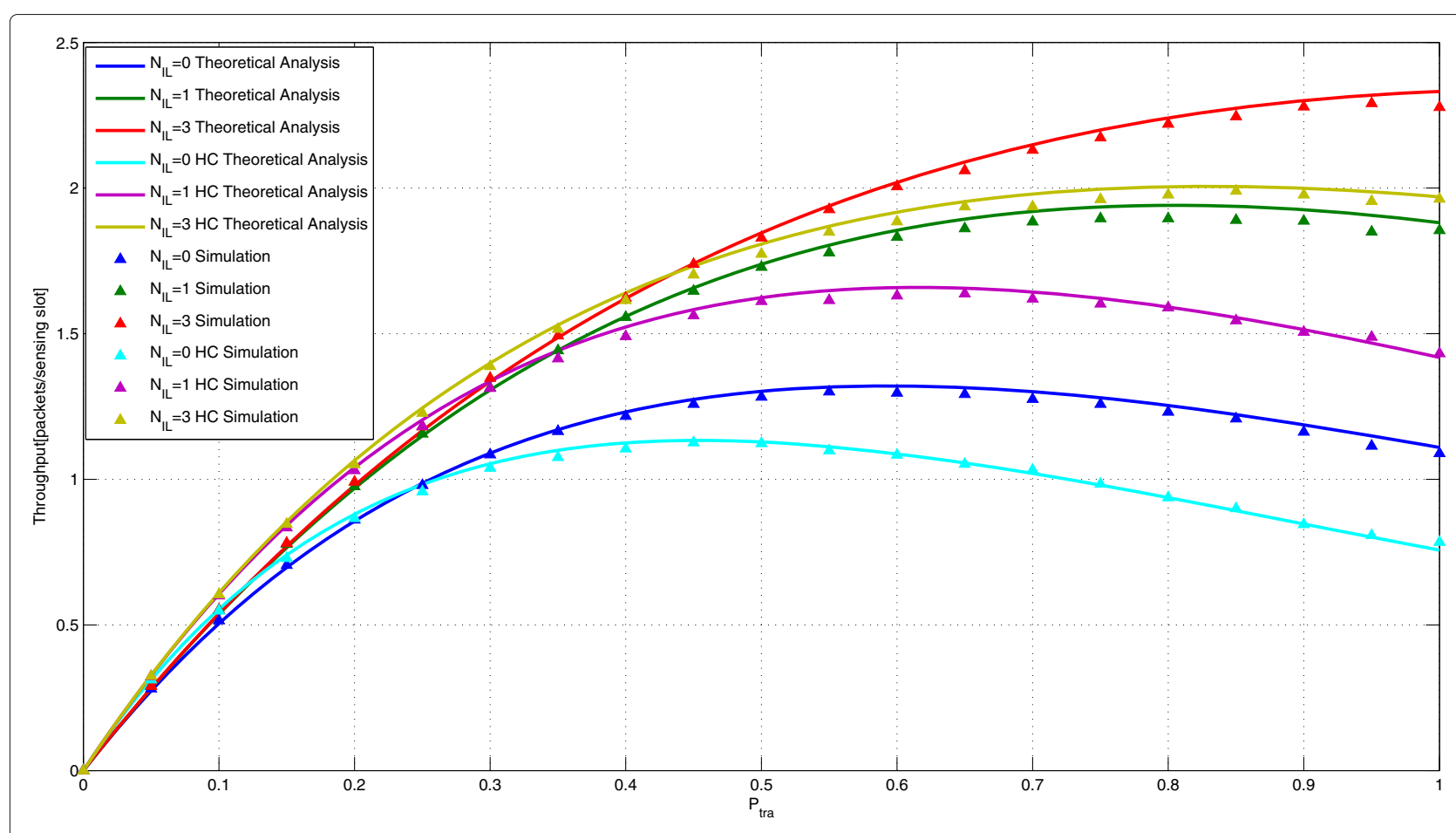

Fig. 8 Throughput comparison versus different sensing schemes

in a low level, but when $P_{\text {tra }}$ is large enough, the sensing method in our model outperforms the "hard combining" scheme.

Figure 9 compares the throughputs in different channel occupancy adopting both sensing methods with imperfect detection. We assume that $\xi=-15 \mathrm{~dB}, N_{\mathrm{IL}}=1$, and $P_{\mathrm{d}}=0.9$. From Fig. 9 , it can be seen that the sensing strategy in this model outperforms the "hard combining" method under any circumstances. In addition, when the channel occupancy rate is high, $q=0.2$ for example, the

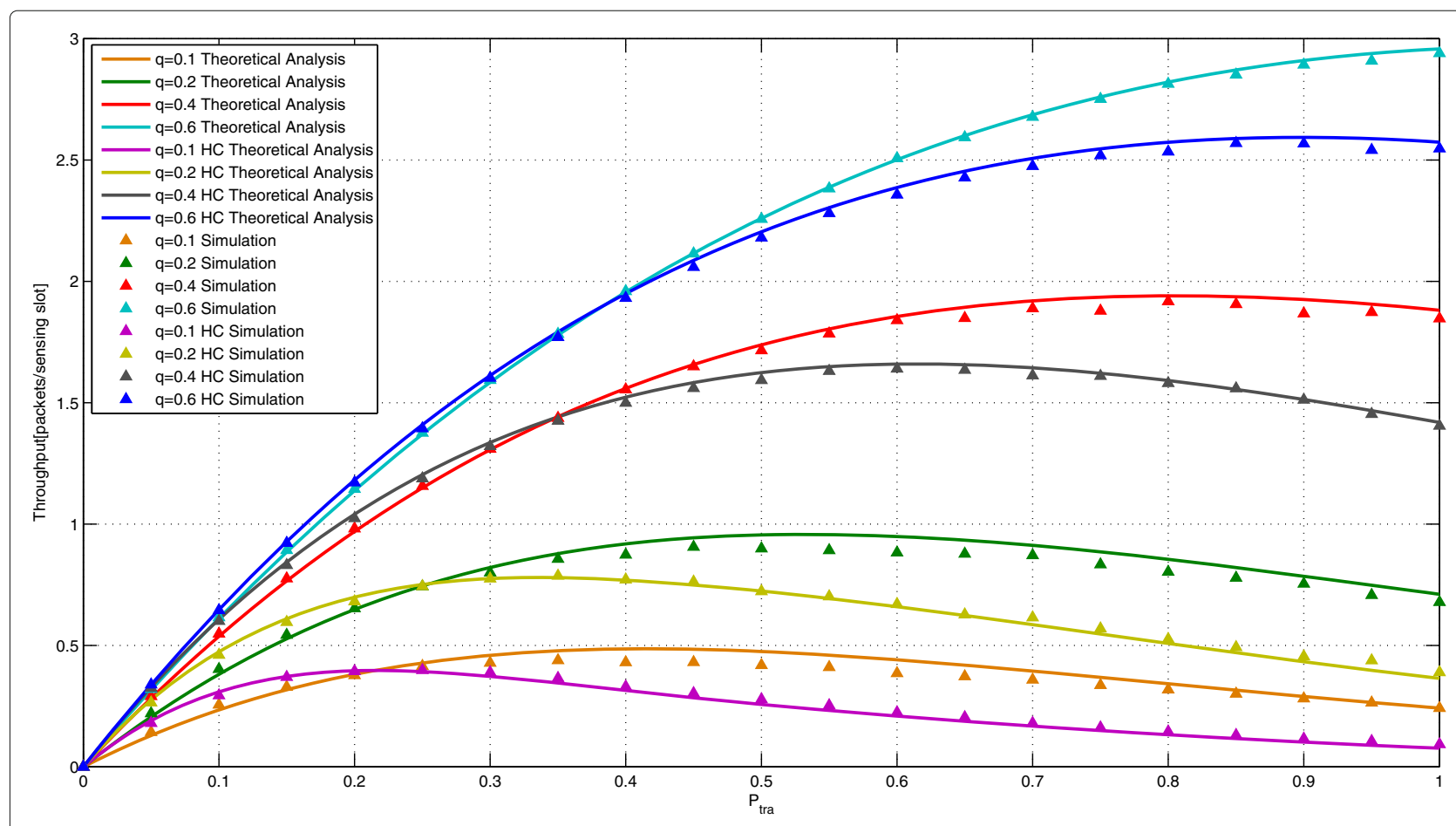

Fig. 9 Throughput with different channel occupancy 
system performs best when $P_{\text {tra }}$ is approximately 0.5 , and satellite earth stations can send the packet once it is generated when channels are not busy, e.g., $q=0.6$. Because all satellite earth stations will get the channel state information at the beginning of transmission phase, it is not a difficult problem for them to adjust $P_{\text {tra }}$ adaptively based on the information to maximize the system throughput.

\section{Conclusions}

In this paper, a spread slotted ALOHA based on multiuser, multi-channel CR model in satellite communication scenario is proposed. In the sensing phase, satellite earth stations will store sampling values on all channels into buffer and then send them to a central cooperator, i.e., a GEO satellite, and the satellite will make a final decision on channel states of all licensed channels. Satellite earth stations will receive the results after the reporting time. Then, satellite earth stations choose an idle channel randomly based on the results and send one packet with a specific probability within a transmission slot. Based on the simulation and numerical analysis, we can draw the following conclusions. First, by comparing the sensing method used in this paper with the traditional "hard combining" sensing scheme, we found that "hard combining" sensing strategy is not as good as the sensing method in this model at the system performance. Second, system throughput improves a lot when SSA scheme is adopted in our model rather than traditional SA strategy. Third, the system will be improved greatly if receiver on the GEO satellite can tolerate more interfering packets which means that the complexity can exchange better performance in our model. Finally, the simulations in different channel occupancy show that the probability that satellite earth stations send a packet can change adaptively to maximize the system throughput; it is not difficult to realize because the satellite will broadcast the channel information at the beginning of transmission phase.

\section{Acknowledgements \\ This work was supported in part by National Natural Science Foundation of China under Grants No.61671183 and 91438205.}

\section{Competing interests}

The authors declare that they have no competing interests.

Received: 27 July 2016 Accepted: 20 September 2016

Published online: 29 September 2016

\section{References}

1. J Mitola, G Maguire, Cognitive radio: making software radios more personal. IEEE Pers. Commun. 6(4), 13-18 (1999)

2. A Hsu, D Weit, CA Kuo, in Wireless Communications and Networking Conference, 2007. Cognitive MAC protocol using statistical channel allocation for wireless ad-hoc networks (IEEE, Hong Kong, 2007), pp. 105-110

3. G Ganesan, et al., Spatiotemporal sensing in cognitive radio networks. IEEE J. Sel. Areas Commun. 26(1), 5-12 (2008)

4. H Hang, et al., in 2012 IEEE 14th International Conference on Communication Technology (ICCT). Throughput maximization for cognitive radio networks with time-domain combining cooperative spectrum sensing (IEEE, Cheng Du, 2012), pp. 235-239

5. $L Y$, et al., Sensing-throughput tradeoff for cognitive radio networks. IEEE Trans. Wirel. Commun. 7(4), 326-1337 (2008)

6. J Yuan, M Torlak, in 7th International Wireless Communications and Mobile Computing Conference. Optimization of throughput and autonomous sensing in random access cognitive radio networks (IEEE, Istanbul, 2011), pp. 1232-1237

7. GX Liu X, M Jia, G Q, Joint cooperative spectrum sensing and channel selection optimization for satellite communication systems based on cognitive radio. Int. J. Satell. Commun. Network (2015). doi:10.1002/sat.1169.

8. Q Zhao, et al., Decentralized cognitive MAC for opportunistic spectrum access in ad hoc networks: a POMDP framework. IEEE J. Sel. Areas Commun. 25(3), 589-600 (2007)

9. Kwon, Sehoon, B Kim, BH Roh, Preemptive opportunistic MAC protocol in distributed cognitive radio networks. IEEE Commun. Lett. 18(7), 1155-1158 (2014)

10. SC Jha, et al., Design of OMC-MAC: an opportunistic multi-channel MAC with QOS provisioning for distributed cognitive radio networks. IEEE Trans. Wirel. Commun. 10(10), 3414-3425 (2011)

11. J Jia, Q Zhang, X Shen, Hc-mac: A hardware-constrained cognitive MAC for efficient spectrum management. IEEE J. Sel Areas Commun. 26(1), 106-117 (2008)

12. GU Hwang, $\mathrm{S}$ Roy, Design and analysis of optimal random access policies in cognitive radio networks. Commun. IEEE Trans. 60(1), 121-131 (2012)

13. X Li, et al., Throughput analysis for a multi-user, multi-channel aloha cognitive radio system. IEEE Trans. Wirel. Commun. 11(11), 3900-3909 (2011)

14. M Hoyhtya, et al., in 2012 IEEE International Symposium on Dynamic Spectrum Access Networks (DYSPAN). Application of cognitive radio techniques to satellite communication (IEEE, Bellevue, 2012), pp. 540-551

15. H Li, Q Guo, Q Li, in 2012 Second International Conference on Instrumentation, Measurement, Computer, Communication and Control (IMCCC). Satellite-based multi-resolution compressive spectrum detection in cognitive radio networks (IEEE, Harbin, 2012), pp. 1081-1085

16. ea Vincenzo Icolari, in 2014 IEEE Global Communications Conference. An energy detector based radio environment mapping technique for cognitive satellite systems (IEEE, Austin, 2014), pp. 2892-2897

17. PVR Ferreira, R Metha, AM Wyglinski, in 2014 IEEE Global Conference on Signal and Information Processing. Cognitive radio-based geostationary satellite communications for ka-band transmissions (IEEE, Atlanta, 2014), pp. 1093-1097

18. D Makrakis, K Murthy, Spread slotted aloha techniques for mobile and personal satellite communication systems. IEEE J. Sel. Areas Commun. 10(6), 985-1002 (1992)

19. S Maleki, et al., Cognitive spectrum utilization in ka band multibeam satellite communications. IEEE Commun. Mag. 53(3), 24-29 (2015)

\section{Submit your manuscript to a SpringerOpen ${ }^{\circ}$ journal and benefit from:}

- Convenient online submission

- Rigorous peer review

- Immediate publication on acceptance

- Open access: articles freely available online

- High visibility within the field

- Retaining the copyright to your article

Submit your next manuscript at springeropen.com 